\title{
Weather Forecasting in India: A Review
}

\author{
Vijay Kumar Didal ${ }^{1}$, Brijbhooshan ${ }^{1}$, Anita Todawat $^{2}$ and Kamlesh Choudhary ${ }^{2}$ \\ ${ }^{1}$ Department of Agronomy, PJTSAU, Hyderabad, Telangana, India \\ ${ }^{2}$ Department of Soil Science, SKNAU, Jobner, Jaipur, Rajasthan, India \\ *Corresponding author
}

\begin{tabular}{|c|c|}
\hline & A B S T R A C T \\
\hline Keywords & \multirow{4}{*}{$\begin{array}{l}\text { Weather forecasting is the application of science and technology to predict } \\
\text { the state of the atmosphere for a given location and they are made by } \\
\text { collecting quantitative data. Soft computing is an innovative approach to } \\
\text { construct computationally intelligent systems that are supposed to possess } \\
\text { humanlike expertise within a specific domain, adapt themselves and learn } \\
\text { to do better in changing environments, and explain how they make } \\
\text { decisions. Soft computing techniques are Fuzzy logic, Neural Network, } \\
\text { Evolutionary computing, Genetic Algorithm etc. In this paper description } \\
\text { about status, scope, types, role, significance, limitations, techniques and } \\
\text { ITKs of weather forecasting in India. }\end{array}$} \\
\hline $\begin{array}{l}\text { Weather forecasting, } \\
\text { Indigenous } \\
\text { Technological } \\
\text { Knowledge (ITK). }\end{array}$ & \\
\hline Article Info & \\
\hline $\begin{array}{l}\text { Accepted: } \\
\text { 07 September } 2017 \\
\text { Available Online: } \\
\text { 10 November } 2017\end{array}$ & \\
\hline
\end{tabular}

\section{Introduction}

Weather forecasting is the prediction of what the atmosphere will be like in a particular place by using technology and scientific knowledge to make weather observations. In other words, it's a way of predicting things like cloud cover, rain, snow, wind speed and temperature before they happen (Cahir, 2013). Weather forecasters use all kinds of tools to achieve this goal. We have instruments called barometers to measure air pressure, radar to measure the location and speed of clouds, thermometers to measure temperature and computer models to process data accumulated from these instruments. However, to this day, humans with good experience can still do a better job at predicting the weather than computer models alone because humans are often involved in picking the most appropriate model for a situation (Craft, 2010).

The main ways the weather can be forecast include looking at current weather conditions, tracking the motion of air and clouds in the sky, finding previous weather patterns that resemble current ones, examining changes in air pressure and running computer models (Banerjee et al., 2003).

The environment in which crops are grown dictates their final yield. Of these environmental factors, climate and weather, the uncontrollable factors have maximum influence on crop productivity (Cahir, 2013). Vagaries of weather subject the crops to 
different ecological situations from year to year leading to differential responses of crops to input use. This situation limits use of costly inputs for realising optimum yield. Therefore, the primary requirement for initiating agronomic measures against weather hazards is foreknowledge of weather situation that is likely to develop in an area (Venkataraman, 2002).

\section{Significance of weather forecasting}

Weather forecasting can help agricultural activities in the following ways:

Planning for necessary inputs during the season

Timely land preparation to take advantage of earliest rain for timely sowing.

Selection of crops and cultivars.

Efficient use of fertilizers.

Predicting pests and diseases incidence for timely action.

Timing of weeds, pests and disease control.

Planning for mitigation adverse effects of weather hazards.

Adjustments in crop harvest timing to reduce the losses at harvest.

\section{Problems of weather forecasting}

The problems of weather forecasting, as seen from the standpoints of mechanics and physics. If, as every scientifically inclined individual believes, atmospheric conditions develop according to natural laws from their precursors, it follows that the necessary and sufficient conditions for a rational solution of the problems of meteorological prediction are the following:
The condition of the atmosphere must be known at a specific time with sufficient accuracy.

The laws must be known, with sufficient accuracy, which determine the development of one weather condition from another.

\section{Forecast requirements during different seasons}

To a large extent, crop production in our country depends on rainfall vagaries. Long range forecasts needed for kharif and rabi are:

\section{Kharif}

Onset and withdrawal of monsoon.

Breaks in monsoon rainfall, and

Occurrence of heavy rainfall.

\section{Rabi}

Rainfall and cold waves during winter.

Onset of heat waves and strong winds in spring, and

Hail storms at commencement of summer.

Organizations involved in weather forecasting

Several organizations all over the world measure weather elements and forecast weather conditions. Accepted norms are developed for measuring, assigning values and codes for different countries. India Meteorological Department was established in 1975 with headquarters at Pune. Agricultural Meteorological Division was started in 1932 for conducting research on crop weather relationships. A major step was taken in the early forties to set up specialized meteorological observatories in crop environment to inculcate weather consciousness among farmers and to develop 
farm environment climatology. This has resulted in steady growth of observatories, besides agromet observatories, synoptic weather stations also record data such as rainfall, temperature, radiation, wind velocity, evaporation, etc. The National Commission on Agriculture recommended establishment of Principal Agromet Observatories in each of the Agricultural Universities.

The synoptic observatories collect information on various weather elements on the basis of which daily forecasts, warnings and weather reports are prepared by five regional forecasting centres situated at Chennai, Nagpur, Mumbai, Delhi and Kolkata. The regional centres also prepare forecast of weather known as weather bulletins indicating the probable date to onset of monsoon, intensity, duration, breaks in rainfall and other adverse weather phenomenon. The bulletins are broadcasted in the regional languages through radio and television along with rural programmes.

\section{Need for and requirements of weather forecasts for agriculture}

\section{Climate-based strategic agronomic- planning}

Weather plays an important role in agricultural production. It has a profound influence on the growth, development and yields of a crop, incidence of pests and diseases, water needs and fertilizer requirements in terms of differences in nutrient mobilization due to water stresses and timeliness and effectiveness of prophylactic and cultural operations on crops. Weather aberrations may cause (i) physical damage to crops and (ii) soil erosion. The quality of crop produce during movement from field to storage and transport to market depends on weather. Bad weather may affect the quality of produce during transport and viability and vigour of seeds and planting material during storage.

Thus, there is no aspect of crop culture that is devoid of the impact of weather. However, (a) the weather requirements for optimal growth, development and yield of crops, incidence, multiplication and spread of pests and diseases and susceptibility to weather-induced stresses and affliction by pests and diseases vary amongst crops, with the same crop with the varieties and with the same crop variety with its growth stages. Even on a climatological basis weather factors show spatial variations in an area at a given time, temporal variations at a given place and year to year variations for a given place and time. For cropping purposes weather over short time periods and year-to-year fluctuations at a place over the selected interval have to be considered. For any given time-unit the percentage departures of extreme values from a mean or median value, called the coefficient of variability, is a measure of variability of the parameter The shorter the time-unit, the greater is the degree of variability of a weather parameter.

Again, intensity of the above three variations differ amongst weather factors. Over short periods of time, rainfall is the most variable of all parameters, both in time and space. In fact for rainfall the short-period inter-year variability is large, which necessitates expressing variability in terms of percentage probability of realizing a given amount of rain or specify the minimum assured rainfall amounts at a given level of probability.

For optimal productivity at a given location crops and cropping practices must be such that while their cardinal phased weather requirements match the temporal march of the concerned weather element(s), endemic periods of pests, diseases and hazardous weather are avoided. In such strategic 
planning of crops and cropping practices, short-period climatic data, both routine and processed (like initial and conditional probabilities), have a vital role to play.

\section{Essentials of weather forecasting}

Essential features of weather forecasting are:

Proper recording of data.

Careful study of synoptic charts.

Search for similar situation from the historical data.

Preparation of the weather condition chart as may be possible in next 24 hours, and

Drawing quick, correct levels and definite conclusions regarding future weather phenomenon.

\section{Elements included in weather forecasting}

From another side, the elements of agricultural weather forecasts vary from place to place and from season to season, but they should refer to all weather elements, which affect farm planning and/or operations, and they ideally would include (WMO, 2001):

Sky coverage by clouds

Precipitation

Temperature (maximum, minimum and dew point)

Relative humidity

Wind Speed and direction

Extreme events (heat and cold waves fog, frost, hail, thunderstorms, wind squalls and gales, low pressure areas, different intensities of depressions, cyclones, tornados, ...)
Bright hours of sunshine

Solar radiation

Dew

Leaf wetness

Pan evaporation

Soil moisture stress conditions and supplementary irrigation for rainfed crops

Advice for irrigation timing and quantity in terms of pan evaporation

Specific information about the evolution of meteorological variables into the canopy layer in some specific cases

Micro-climate inside crops in specific cases.

\section{Types of weather forecasting}

Based on time or duration of forecasting period, the weather forecasting can be divided into six categories:

Now-casting (NC)

Very short range weather forecasting

Short range weather forecasting

Medium range weather forecasting

Extended range weather forecasting

Long range weather forecasting

\section{Now-casting (NC)}

Current weather variables and 0-6 hour's description of forecasted weather variables. A relatively complete set of variables can be produced (air temperature and relative humidity, wind speed and direction, solar radiation, precipitation amount and type, cloud). Prerequisite is the operational continuity and the availability of an efficient broadcasting systems (e.g. very intense 
showers affecting a given territory must be followed with continuity in provision of information for final users). Accuracy is very high and potential usefulness is low (Table 1 ).

\section{Very short range weather forecasting}

Up to 12 hours description of weather variables. A relatively complete set of variables can be produced (air temperature and relative humidity, wind speed and direction, solar radiation, precipitation amount and type, cloud). Prerequisite is the availability of an efficient broadcasting systems (e.g. frost information must be broadcasted to farmers that can activate irrigation facilities or fires or other systems of protection). Accuracy is very high and potential usefulness is moderate.

\section{Short range weather forecasting}

Short range weather forecasts are for a period of 12 hours to 72 hours. These daily forecasts are useful to irrigation engineers and farmers. A relatively complete set of variables can be produced (air temperature and relative humidity, wind speed and direction, solar radiation, precipitation amount and type, cloud). In SRF the attention is centred on meso scale features of different meteorological fields. SRF can be broadcasted by a wide set of media (newspapers, radio, TV, web, etc.) and can represent a fundamental information for farmers. Accuracy and potential usefulness are high.

\section{Agricultural applications of short range weather forecasting}

Timing of field operations.

Soil workability.

Drying rate of soil.

Irrigation scheduling.

Spray applications.
Insect disease effects.

Livestock protection from cold and heat.

\section{Medium range weather forecasting}

Medium range weather forecasts are for periods of 3 to 10 days. A relatively complete set of variables can be produced (air temperature and relative humidity, wind speed and direction, solar radiation, precipitation amount and type, cloud). In MRF the attention is centred on synoptic features of different meteorological fields.

MRF can be broadcasted by a wide set of media (newspapers, radio, TV, web etc.) and can represent a fundamental information for farmers. Accuracy is high or moderate until 5 days; lower after and potential usefulness is very high.

\section{Agricultural applications of short range weather forecasting}

Determine depth of sowing for optimal seedling emergence.

Decide whether to sow or not.

Plan irrigation based on expected rainfall.

Ensure maximum efficiency of spraying.

Decide to harvest or not to harvest.

Management of labour and equipment.

Plan for animal feed requirement.

Livestock protection from cold and heat.

\section{Extended range weather forecasting}

Extended range weather forecasts are for periods of 10 to 30 days. Forecast is usually restricted to Temperature and precipitation. 


\section{Long range weather forecasting}

The long range weather forecasts are issued thrice in year. Validity period of long range weather forecast is 10 to 30 days. The long range forecasts are useful for choosing cropping patterns.

\section{Agricultural Applications of long range weather forecasting}

Crop Planning - Marginal crops Vs Normal Crops

Choose crop varieties to suit the expected weather

Determine expected crop yield

Plan area to be cultivated to get the required crop produce

\section{Methods of weather forecasting}

The nature of modern weather forecasting is not only highly complex but also highly quantitative. The various methods used in forecasting the weather are as follows:

Synoptic weather forecasting,

Numerical methods, and

Statistical methods.

\section{Synoptic weather forecasting}

The first method is the traditional approach in weather prediction. This primary method continued to be in use until the late 1950s. Synoptic" means that the observation of different weather elements refers to a specific time of observation. Thus, a weather map that depicts atmospheric conditions at a given time is a synoptic chart to a meteorologist. In order to have an average view of the changing pattern of weather, a meteorological centre prepares a series of synoptic charts every day.
Such synoptic charts form the very basis of weather forecasts.

As stated earlier, the task of preparing synoptic charts on a regular basis involves huge collection and analysis of observational data obtained from thousands of weather stations. From the careful study of weather charts over many years, certain empirical rules were formulated. These rules helped the forecaster in estimating the rate and direction of the movement of weather systems.

Synoptic methods involved detailed analysis of current weather reports from a large area. The current weather patterns are related with the past analogous situations and forecasts are prepared on the assumption that a current weather situation will behave on the lines of the past analogous situations.

Often selection of the past analogous situations is based on the experience and memory of the forecast, but with the advent of computers, picking analogues has become faster and more objective. This method is useful for short range forecasts.

\section{Numerical Weather Prediction (NWP)}

Uses the power of computers to make a forecast. Complex computer programs, also known as forecast models, run on supercomputers and provide predictions on many atmospheric variables such as temperature, pressure, wind, and rainfall. A forecaster examines how the features predicted by the computer will interact to produce the day's weather.

The NWP method is flawed in that the equations used by the models to simulate the atmosphere are not precise. If the initial state is not completely known, the computer's prediction of how that initial state will evolve will not be entirely accurate. 
In this technique the behaviour of atmosphere is represented by equations based on physical laws governing air movement, air pressure and other information. This technique is found suitable for medium range forecasts. Accurate meteorological data coupled with modern weather prediction techniques and transmission of data through telemetric network with suitable backing agricultural support systems, will imply a sea-chamber in ability to plan agriculture in an optional way for a given set of weather conditions.

\section{Statistical methods}

Statistical methods are used along with the numerical weather prediction. This method often supplements the numerical method. Statistical methods use the past records of weather data on the assumption that future will be a repetition of the past weather. The main purpose of studying the past weather data is to find out those aspects of the weather that are good indicators of the future events. After establishing these relationships, correct data can be safely used to predict the future conditions. Only overall weather can be predicted in this way. It is particularly of use in projecting only one aspect of the weather at a time. At macro level, weather forecasting is usually done using the data gathered by remote sensing satellites. Weather parameters like maximum temperature, minimum temperature, extent of rainfall, cloud conditions, wind streams and their directions, are projected using images taken by these meteorological satellites to assess future trends.

The satellite-based systems are inherently costlier and require complete support system. Moreover, such systems are capable of providing only such information, which is usually generalized over a larger geographical area. The variables defining weather conditions like temperature (maximum or minimum), relative humidity, rainfall etc., vary continuously with time, forming time series of each parameter and can be used to develop a forecasting model either statistically or using some other means like artificial neural networks.

Regression equations or other sophisticated relationships are established between different weather elements and the resulting climate. Normal selection of predictors of weather parameters is based on possible physical relationship with the predictant. These techniques are useful for short as well as for long range forecasting. Multiple regression equation developed to provide annual rainfall based on 16 parameters is quite successful in India.

Table.1 Accuracy and usefulness of weather forecasting for agriculture

\begin{tabular}{|l|l|c|c|}
\hline S. No. & Forecast type & Accuracy & Potential usefulness \\
\hline 1. & Now-casting (NC) & Very high & Low \\
\hline 2. & Very Short Range Forecast (VSRF) & Very high & Moderate \\
\hline 3. & Short Range Forecast (SRF) & High & High \\
\hline 4. & Medium Range Forecast (MRF) & High & Very high \\
\hline 5. & Extended Range Forecast (ERF) & Moderate & Poor \\
\hline 6. & Long Range Forecast (LRF) & Very low & Poor \\
\hline
\end{tabular}


Table.2 Terms used in rainfall forecasting

\begin{tabular}{|l|c|}
\hline Descriptive Term used & Rainfall amount in mm \\
\hline No Rain & 0.0 \\
\hline Very light Rain & $0.1-2.4$ \\
\hline Light Rain & $2.5-7.5$ \\
\hline Moderate Rain & $7.6-35.5$ \\
\hline Rather Heavy & $35.6-64.4$ \\
\hline Heavy Rain & $64.5-124.4$ \\
\hline Very Heavy Rain & $124.5-244.4$ \\
\hline Extremely Heavy Rain & \multicolumn{1}{|c|}{$>244.5$} \\
\hline Exceptionally Heavy Rain & $\begin{array}{l}\text { When the amount is a value near about the highest } \\
\text { recorded rainfall at or near the station for the month } \\
\text { or season. However, this term will be used only } \\
\text { when the actual rainfall amount exceeds } 12 \text { cm. }\end{array}$ \\
\hline
\end{tabular}

Table.3 Weekly/seasonal rainfall distribution on regional scale

\begin{tabular}{|l|c|}
\hline \multicolumn{1}{|c|}{ Category } & Percentage departure of actual rainfall from normal rainfall \\
\hline Excess Rainfall & $+20 \%$ or more \\
\hline Normal Rainfall & $-19 \%$ to $+19 \%$ \\
\hline Deficient Rainfall & $-20 \%$ to $-59 \%$ \\
\hline Scanty Rainfall & $-60 \%$ to $-99 \%$ \\
\hline No rain & $-100 \%$ \\
\hline
\end{tabular}


Fig.1 The Advanced Weather Interactive Processing System (AWIPS)

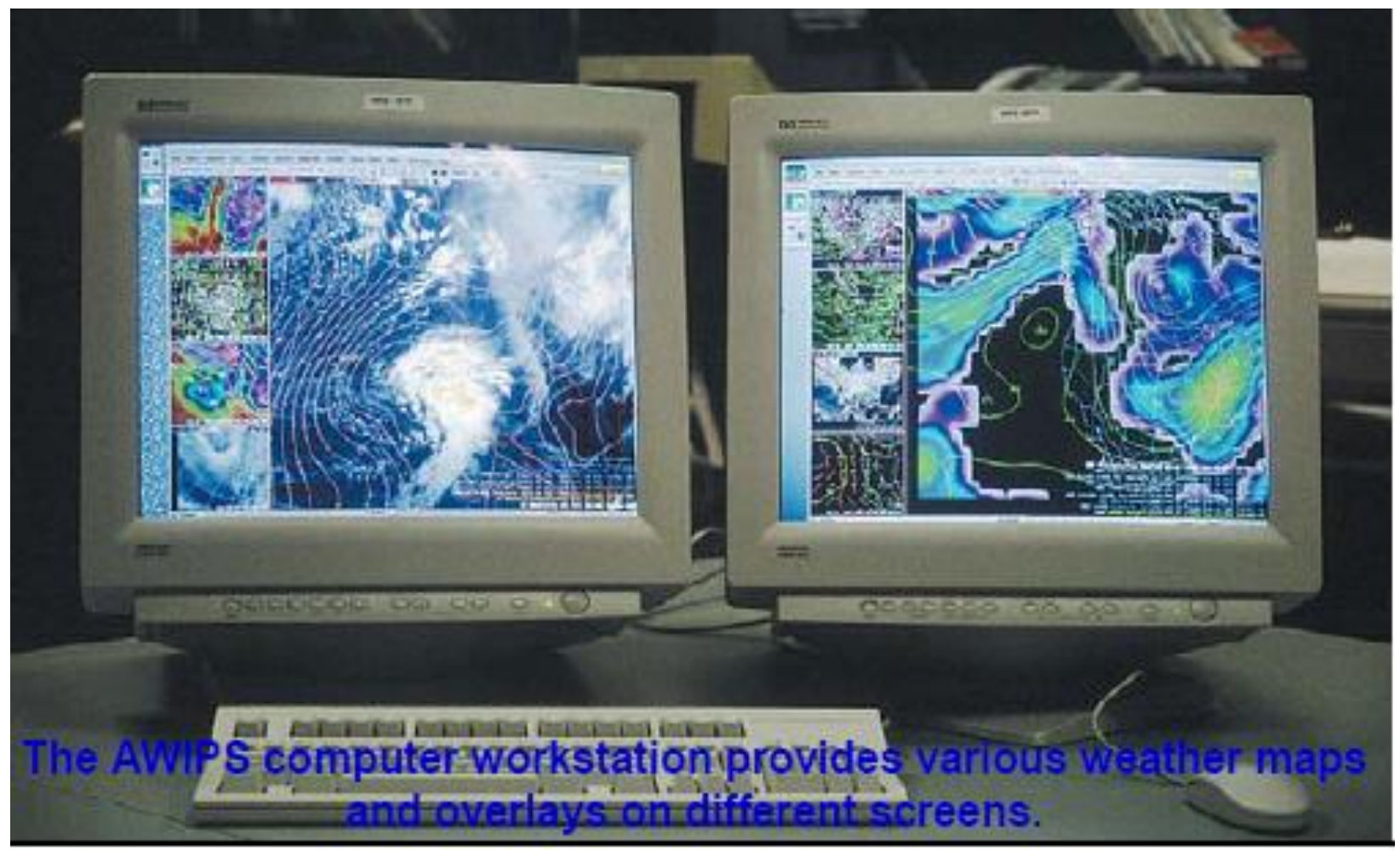

Fig.2 Disseminating weather information through different agencies

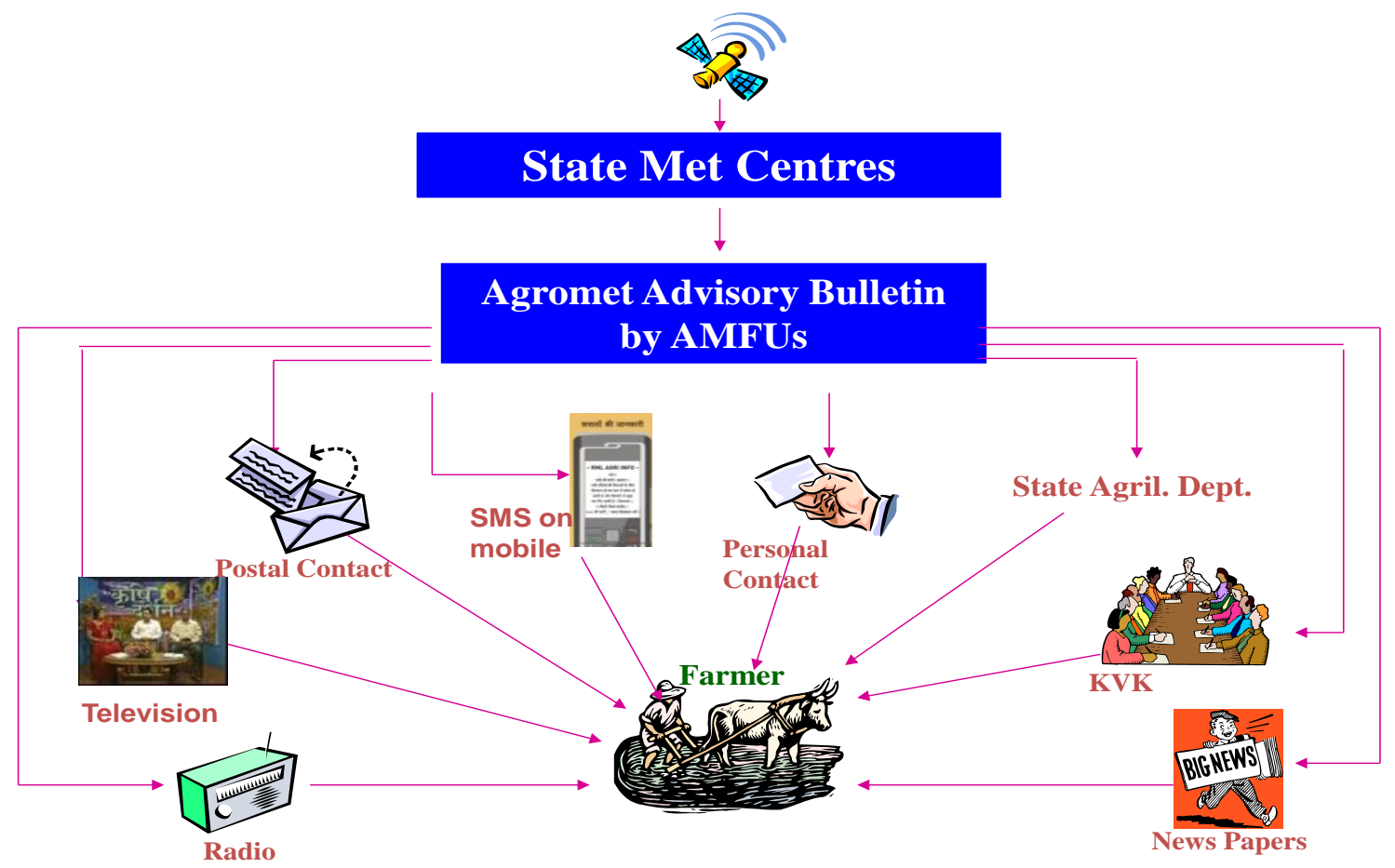


Fig.3 District wise correct and usable rainfall forecast (\%) in different districts of Telangana during kharif, 2015

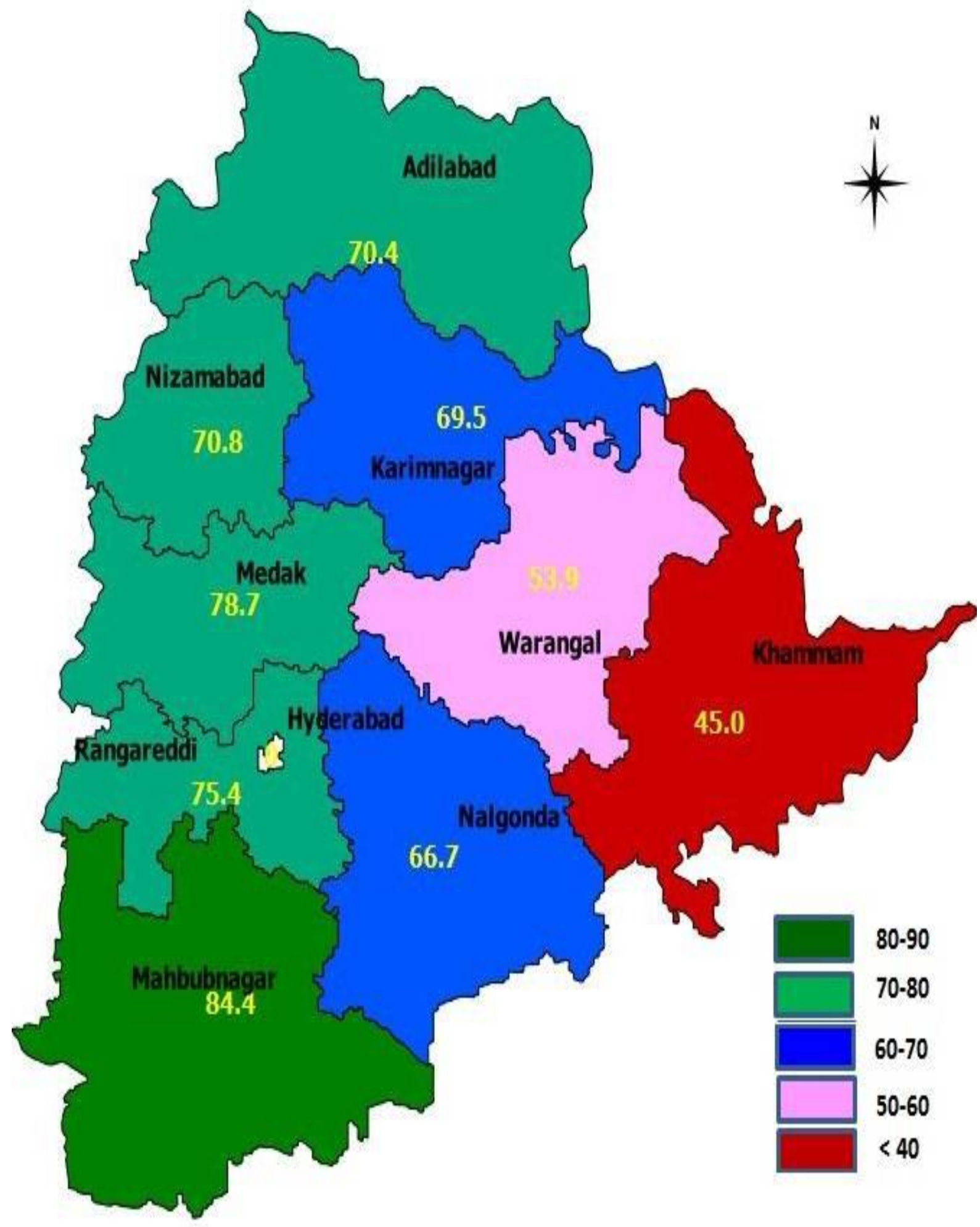




\section{Advanced Weather Interactive Processing System (AWIPS)}

The Advanced Weather Interactive Processing System (AWIPS) computer workstation provides various weather maps and overlaps on different screens. AWIPS works in four steps which are given below:

Data communication.

Storage.

Processing, and

Display.

\section{ITK for weather prediction}

Out of various the factors which control agricultural production, weather is the only factor over which man has no control and hence it has an overwhelming dominance over the success or failure of agricultural enterprise. It is an accepted fact that food production is inextricably linked with climate and weather. It is also reported that weather induced variability of food production is more than 10 per cent. This variability can be as high as 50 per cent of the normal production in respect of smaller areas situated in arid and semi-arid regions. In order to reduce risks of loss in food production due to the vagaries of weather, weather per se, should be taken into account as one of the major inputs in agricultural planning. That is why forecast of weather parameters play a vital role in agricultural production. It also aids in minimize crop losses to a considerable extent. Thus development and refinement of the art of weather prediction has been essential since time immemorial. In present times we have many improved technologies for making weather forecasts as well as for their dissemination. Previously when there was no such technology available farmers based their prediction on many natural, cultural and social phenomena. Some of these are discussed below:
Visible spectrum around the sun and the moon

People predicted weather after observing the visible spectrum around the sun or moon. If the spectrum around the sun had a greater diameter than that around the moon, they predicted rainfall after a day or two.

Some people based their weather prediction on the nature of the solar halo, specifically: "if the spectrum around the sun has a larger diameter then rainfall is assured.

All the photometers are a luminous phenomenon produced by the reflection, refraction, diffraction or interference of light from the sun or moon. The visible spectrum of light around the sun or moon is called halo, or carona according to its distance from the sun or moon.

If the distance is more then it is called the halo phenomenon, which is caused by a layer of thin veil of cirrus clouds i.e. non rain bearing clouds. But if the distance is less, it is called corona phenomena produced by somewhat dense clouds which may cause rainfall. The accuracy of this indigenous observation can be as high as 50 per cent.

\section{Cloud and wind direction}

If there is an accumulation of clouds in the South-East direction in a layered form accompanied by winds blowing from the southern direction then it is claimed that there will be rainfall within a day or two.

\section{Weather prediction through birds and other animals}

Farmers also predict weather by observing closely the different activities of various birds, animals etc. The following are some indigenous beliefs: 
It is believed that on a hot summer day the cry of the bird called "Nialu" for water brings rainfall

During the rainy season farmers observe the "Matilari" bird (House swift) and they predict heavy rainfall if the bird flies high in the sky

If the "Maina" bird bathes in the water it indicates that there will be rainfall within one or two days

During long hot days in summer if the cry of theapiha bird is heard then people believe that God will quench her thirst and there will be rainfall after one or two days.

A group of sparrows frolicking in the sand indicates that there will be rainfall that day or the next day and if they are observed to be playing in water then it is believed that the weather will be dry for some days to come.

If the "Jonks" (Leechs) are immobile/stationary at the water surface (Pond) then dry weather is predicted but if they move rapidly in the upward and downward direction in water then rainfall is predicted.

If the "Tatihari" bird (Lapwing) lays her eggs on the higher portion of the field then heavy rainfall is predicted during the coming rainy season but if the eggs are laid in the lower portion of the field then a drought is predicted. These birds never construct a nest but lay their eggs on bare soil.

Further it is also believed that if a single egg is laid, then there will be rainfall only for one month out of four months of the rainy season.

If two eggs are laid then rainfall will occur for two months and similarly four eggs indicate there will be rainfall during all the four months of the rainy season.
If there is a swelling on the lower portion of the camel's legs then rainfall is predicted by the farmers. The swellings are probably caused due to higher relative humidity.

If the "Tillbohara" (Dragon fly), which appears generally in the rainy season, are observed to swarm in a large group over a water surface (Pond) then dry weather is predicted but if they swarm over open dry lands or fields then early rainfall is predicted by the farmers.

If the colour of the clouds is similar to the colour of the wings of the Titar bird (Partridge) i.e. grey or black-grey and strong eastern winds are also blowing then assured rainfall is predicted by the farmers.

The clouds of a colour similar to that of the said bird are rain bearing clouds i.e. of cumulonimbus type.

If centipedes emerge from their holes carrying their eggs in swarms in order to shift them to safer places (within the house) then farmers predict early rainfall The centipedes do this so as to avoid egg damage which can be caused by rain water.

When spider nets are plentiful on grasses, sticks of tomato crop and on trench bean crop then it is estimated that the rainy season is over.

\section{Social and cultural beliefs}

Many cultural, social and religious beliefs and activities superstitious pertaining to the prediction of future weather prevail since generations. From time immemorial farmers have predicted the weather on the basis of these beliefs/activities.

The following are some examples from the western Himalayan region. 
If the first 10-15 of the month "Jeth" (MayJune) are very hot then good rainfall/monsoon is predicted during the ensuing rainy season. This results probably from the low pressure zone in north-west India that is generated due to the high temperatures.

The Soolini Mela (Festival) is organised in Solan, during the month of June every year. People of this area firmly believe that rainfall will occur on the very day of the festival or one day before or day after the festival

It is also believed, that when grey coloured clouds descend below the hill tops then they definitely cause rainfall.

If the "Khejri" tree bears good fruit in a particular year then farmers predict good rainfall during the next rainy season and vice versa less rain is predicted in the event of a poor fruit crop.

If the Chakkala-Belan, (rolling pin and board), used in the Kitchen, show moisture on them then within few days rainfall is expected.

In villages elderly farmers usually carry a small bag for "Tambaku" (Tobacco) for Hukka (Smoking device). When this bag shows more moisture in the Tambakku then farmers predict rainfall within one or two days.

\section{Some folk-lore regarding weather forecasting}

The folk-lore of the popular poet Gag and his wife Bhahdari, who lived during the 17th century, regarding weather forecasting are still very popular in northern India. Some are given as under:

When strong eastern winds blow continuously then it is estimated that the rainy season has come.
When days are very hot and there is dew at night, then according to Gag, there are very limited chances of rainfall.

When cloudy days are accompanied by clear nights and the eastern winds blow somewhat strongly, then according to Gag no rainfall is predicted. Thus there is accompanied by a shortage of water in ponds, rivers etc. Consequently clothes are washed using water from wells.

When a rainbow is formed in the direction of Bengal then there will be rainfall, if not by the evening then definitely by next morning.

During the rainy season, if a cloud appears on Friday and Saturday then rainfall is predicted either for Sunday or Monday.

\section{National agromet advisory bulletin}

In order to provide direct services to the farming community of the country an exclusive Division of Agricultural Meteorology was set up in 1932 under the umbrella of Dissemination of Agromet Advisory in India.

Under the project, advisories are primarily disseminated to farmers by mass mode, outreach at village level and human face for advisory dissemination. Advisories are being disseminated to farmers through following the multi-channel system: All India Radio (AIR) and doordarshan, private $\mathrm{TV}$ and radio channels, Mobile phones/SMS, newspapers, internet, virtual academy/virtual universities/NGOs, Kisan Call Centres/ICAR and other related Institutes/Agricultural Universities/Extension network of state/central Agriculture Departments, Krishi Vigyan Kendras.

Now-casting have very high accuracy and medium range weather forecasting have very high potential usefulness than the others. 
Advanced Weather Interactive Processing System (AWIPS) is a computer based system which provides fastest and accurate data on future weather conditions. In villages of India Indigenous Technological Knowledge (ITK) is used for weather forecasting.

\section{References}

Banerjee, S. K., Chattopadhyay, N. and Das, H. P., 2003, Study of weather-based agricultural folklore of West Bengal. Pre-Published Sci. Rept. No.1, I.M.D.

Basu, S., 1953, Weather Lore in India. Ind. J. Meteorol. Geophys., 4: 3-12.

Brown, L. H. and Cocheme, J., 1973, A study of the agroclimatology of the highlands of eastern Africa. WMO Technical Note No. 125. WMO No. 339, Geneva.

Cahir J. J., 2013, Weather Forcasting. Encyclopedia Britannica. Accessed on June 2013 (http://www.britannica.com/EBchecked/ topic/638321/weather-forecasting).

Cocheme, J. and Franquin, P., 1967, An agroclimatology survey of a seisrid area in Africa, south of the Sahara. WMO Technical Note No. 86. WMO No. 210.TP.110, Geneva.

Craft E. D., 2010, An economic history of weather forecasting. The Economic History Association (EH.net). Accessed on June 2013 (http://eh.net/encyclopedia/article/craft. weather.forcasting.history).
Hagedorn, R., Doblas-Reyes, F. J. and Palmer, T. N., 2005, The rationale behind the success of multi-model ensembles in seasonal forecasting. $I$. Basic concept. Tellus, A 57: 219-233.

Harrison, M., 2005, The development of seasonal and inter-annual climate forecasting. Climatic Change, 70: 201220.

Stigter, C. J., 2006, Agro-meteorological services in various parts of the world, under conditions of a changing climate. Austin Bourke Memorial Lecture, Dublin. Extended Abstract available on the INSAM web site (www. agrometeorology. org) under "Accounts of operational agrometeorology".

Venkataraman, S., 2001, A simple and rational agroclimatic method for rainfall zonations in dryland areas. Ind. $J$. Environm. Ecoplan., 5: 135-144.

Venkataraman, S., 2002, Tabular aids for computation of derived agro meteorological parameters on a weekly basis. J. Agrometeorol., 4: 1-8.

Vogel, C., O’Brien, K., 2006. Who can eat information? Examining the effectiveness of seasonal climate forecasts and regional climate-risk management strategies. Climate Res., 33: 111-122.

WMO, 2001. Scope of public weather services data and products. WMO TD No. 1054, Geneva.

\section{How to cite this article:}

Vijay Kumar Didal, Brijbhooshan, Anita Todawat and Kamlesh Choudhary. 2017. Weather Forecasting in India: A Review. Int.J.Curr.Microbiol.App.Sci. 6(11): 577-590. doi: https://doi.org/10.20546/ijcmas.2017.611.070 